\title{
Estacionalidad y variación circadiana del número de muertes en México
}

\author{
Rogelio Pérez-Padilla, ${ }^{*} \bowtie$ David Martínez-Briseño, * Rosario Fernández-Plata,* \\ Ramón González-Camarena, Luis Torre-Bouscoulet*
}

*Instituto Nacional de Enfermedades Respiratorias Ismael Cosío Villegas, Ciudad de México; *Universidad Autónoma Metropolitana. Trabajo recibido: 29-IX-2017; aceptado: 03-XI-2017

\begin{abstract}
RESUMEN. Varias enfermedades ocasionan fallecimientos con periodicidad estacional y algunas pueden tener periodicidad circadiana. EI objetivo del trabajo fue identificar periodicidades en el número de fallecimientos en México. Se analizaron los certificados de defunción entre 2000 y 2010, con la décima versión de la Clasificación Internacional de Enfermedades (CIE-10), registrando la fecha y hora de muerte y seleccionando los siguientes padecimientos frecuentes: enfermedad pulmonar obstructiva crónica (EPOC), neumonía e influenza, insuficiencia cardíaca, cardiopatía izquierda (isquémica e hipertensiva), neoplasias malignas y diabetes mellitus (DM) y las causas restantes de muerte. Se buscaron asociaciones entre el número de fallecimientos y la hora, mes y año de defunción mediante modelos de regresión de Poisson, con variables indicadoras para hora, mes y año. Resultados: La neumonía e influenza tuvieron el patrón estacional (invernal) con mayor claridad y amplitud, pero también lo tuvieron la insuficiencia cardíaca, la cardiopatía izquierda, la EPOC y la DM, pero no las muertes perinatales. Observamos un patrón circadiano en el número de fallecimientos con un máximo entre las 7-11 a.m. y otro de menor amplitud cerca de las 8 p.m. en las enfermedades estudiadas salvo las perinatales. Conclusiones: La estacionalidad de las muertes analizadas probablemente se deba a los brotes de influenza y, si es así, son potencialmente prevenibles con vacunación. El mecanismo del incremento matutino en varios de los fallecimientos se desconoce, aunque se ha considerado secundario al incremento en actividad simpática relacionada con el estado alerta y el inicio de la actividad física.
\end{abstract}

Palabras clave: México, muertes, certificación, estacionalidad, ciclo circadiano.

ABSTRACT. Several diseases cause deaths with seasonal periodicity and some may have circadian periodicity. The objective was to identify periodicities in the number of certified deaths in Mexico. We analized death certificates from 2000 to 2010, with the tenth version of the International Classification of Diseases (ICD-10), including date and hour of death and selecting frequent respiratory and general causes of death: chronic obstructive pulmonary disease (COPD), tuberculosis, pneumonia-influenza, heart failure, left heart disease (hypertensive and ischemic), malignant tumors and diabetes mellitus (DM) and the remaining causes of death. We searched for associations between the number of deaths, and the hour, month and year of death, through Poisson regression models, including indicator variables for hour, month and year. Results: Pneumonia and influenza deaths had a marked seasonal pattern (peak in winter), but the pattern was also observed for heart failure, left heart disease, COPD and DM but not in perinatal deaths. We observed a circadian pattern in deaths with a maximum between 7-11 AM, and a second peak about 8 PM in the studied diseases except by perinatal causes. Conclusions: Seasonality of analyzed deaths is likely due to influenza and if this is true, could be preventable with vaccination. The mechanism of diurnal increase in deaths is unknown although usually attributed to increase in symphathetic activity associated to awakening and start of physical activity.

Key words: Mexico, deaths, death certificates, seasonality, circadian rhythm.

\section{INTRODUCCIÓN}

Muchos de los fenómenos biológicos ocurren cíclicamente. Uno de los casos más conocidos es la estacionalidad de las infecciones respiratorias, en particular de la influenza, que se incrementa sustancialmente en los inviernos de los países con estaciones más o menos diferenciadas. ${ }^{1}$ Las muertes atribuibles a influenza, por lo mismo, se incrementan en invierno y bajan en verano, y tienen un comportamiento cíclico estacional. Varias otras causas de muerte siguen la estacionalidad de la influenza y probablemente se deban a la misma, con una certificación mal asignada. Por ejemplo, las muertes generales, las cardiovasculares y las respiratorias en general, se incrementan en la etapa invernal y dentro de estos grupos las muertes que exceden un patrón basal invernal se atribuyen a la influenza. ${ }^{1}$ 
Adicionalmente, pueden existir variaciones del número de muertes de acuerdo a la hora del día, con un ciclo de 24 horas o circadiano. ${ }^{2-12}$ Éstas se han reportado en algunas muertes cardiovasculares, y aunque los motivos del ritmo no están completamente aclarados, podrían considerarse los cambios en el ciclo sueñovigilia o reposo-actividad, las oscilaciones en el control del sistema nervioso autónomo, o de las secreciones hormonales, o una combinación de los anteriores.

El objetivo de nuestro trabajo fue identificar oscilaciones estacionales y circadianas en el número de muertes en México entre 2000 y 2010 para algunos de los padecimientos respiratorios y cardiovasculares más comunes; es decir, explorar si se encuentran meses u horas en los cuales se incrementen los fallecimientos. Encontrar ritmos en las muertes, puede generar hipótesis sobre posibles factores causales y adicionalmente potenciales medidas preventivas.

\section{MÉTODOS}

Se conjuntaron las bases de datos de certificación de muertes en México entre 2000 y 2010, con un total de $5,346,186$ muertes, todas codificadas de acuerdo con la décima clasificación internacional de enfermedades (CIE-10).

Debido al interés en analizar el impacto de la hora de muerte, pero también las contribuciones de estacionalidad o mes del año y año de muerte en el riesgo de incrementar número de muertes, se eliminaron los registros que carecían de esta información. La base de datos incluía para cada registro, año, mes, día y hora de la muerte (sin fracciones de hora).

Con el objetivo de contar con registros de muerte en la mayoría de las horas evaluadas, se seleccionaron solamente padecimientos o grupos de padecimientos, agrupados de una manera estandarizada ${ }^{13}$ que contribuyeran a un número significativo de muertes ya sea respiratorias: enfermedad pulmonar obstructiva crónica (EPOC, códigos J40-J44), tuberculosis y sus secuelas (A15-A19, B90, A31), enfermedades pulmonares intersticiales primarias y secundarias (J60-J80, J82-J84, J99.0, M05-M14, M30-M36), neumonía e influenza (J10-J18), cáncer pulmonar (C34), pero también otras causas comunes de muerte como cardiopatías del lado izquierdo del corazón (I20-I25, I10-I13), insuficiencia cardíaca (IC, I50), muertes perinatales (P0-P96), diabetes mellitus (E10-E14), muertes por neoplasias malignas (C0-C97 sin la C34, y D0-D9, D37-D48) y el conjunto del resto de causas de muerte.

La base de datos se completó hora por hora, llenando con ceros las horas faltantes en los registros originales (las horas sin muertes tenían el dato faltante, que se sustituyó con cero muertes). Para hacer las bases de datos más manejables para el análisis se separaron para cada una de las enfermedades.

Con el fin de explorar la asociación del número de muertes con hora del día, mes, estación y año se tomaron varias estrategias.

1. Análisis exploratorio: se concentraron para cada una de las enfermedades analizadas todas las muertes acaecidas durante los 10 años en: a) cada una de las horas del día, b) cada uno de los meses del año; con esta estrategia pueden observarse en forma gráfica y numérica cambios en la frecuencia de muertes. Para hacerlas comparativas en enfermedades con diferente número de muertos, se graficaron en forma porcentual; es decir, para explorar el impacto de los meses se graficó el porciento de muertes ocurriendo en cada mes y así para los años y las horas. De no existir una asociación entre el mes y el número de muertes para un padecimiento en particular, se esperaría uniformidad en el porcentaje de muertes en cada mes, o en cada hora del día.

2. Como parte del análisis exploratorio se hicieron gráficas como series de tiempo, que incluyen el número de muertes en el eje vertical y el tiempo en el horizontal, lo que permite ver alteraciones recurrentes. Adicionalmente un análisis de autocorrelación permite ver periodicidades.

3. Utilizando modelos de regresión de Poisson, se analizaron para cada una de las enfermedades seleccionadas, la asociación del número de muertes y de la hora del día (figuras 1 y 2 ), del mes del año (figura 3) y del año de muerte (figura 4), por medio de variables indicadoras (dummy): es decir se incorporaron en los modelos 24 variables con las horas de muerte, 12 variables con el mes del año y 10 con el año de muerte dejando en los modelos una de cada grupo fuera para ser la comparativa (en general mes 1, año 1, y hora 1). Como las horas evaluadas en los años compilados son $>96,000$ casi todos los coeficientes de los modelos de regresión tienen un error estándar muy pequeño y resultan estadísticamente significativos al nivel de $p<0.05$. También es importante reconocer que excepto para las causas más comunes de muerte, en el análisis horario hay numerosos ceros, es decir, horas sin fallecimientos.

- Los modelos de regresión de Poisson se realizaron de la manera estándar, y además modelos con características autorregresivas, ya que las cuentas de muertos de un día no son independientes de las del siguiente o expresado en forma alternativa, si se considera que puede haber estaciones de año, meses, u horas del día con 
más muertes por un ciclo biológico, las cuentas de muertos de dos unidades de tiempo contiguas serán más parecidas que dos separadas y por lo tanto no son independientes. Se registró el reporte del cociente de tasa de incidencia (incidence rate ratio $I R R$ ) y se utilizaron los coeficientes para hacer gráficas relacionando el IRR con la hora, mes o año. Los modelos reportados incor-

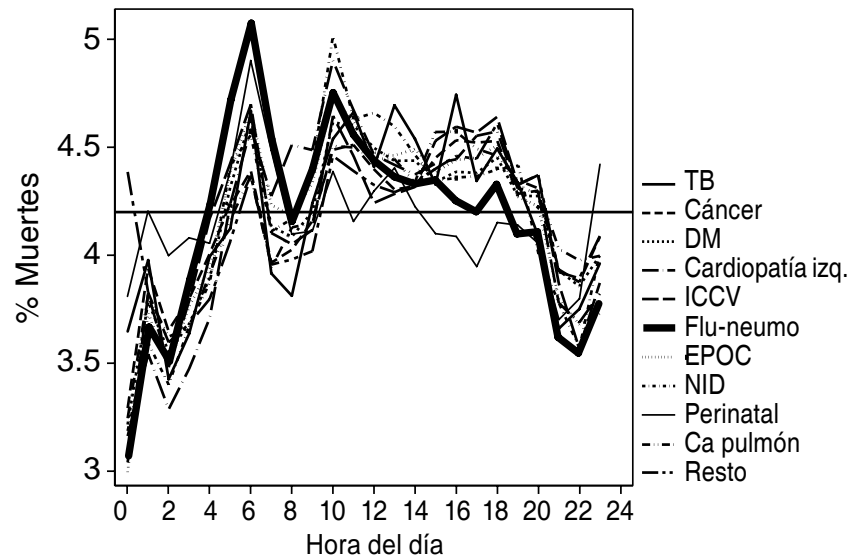

Figura 1. Muertes por hora del día, acumulativo de $2000 \mathrm{~A}$ 2010. Se ve en el eje vertical el porciento de muertes que ocurre en cada hora del día para variadas enfermedades. Si la distribución fuera aleatoria, correspondería a cada hora $4.16 \%$ de las muertes (línea horizontal), sin embargo, en general se incrementan las muertes a las 7 a.m. y a las 11 a.m.

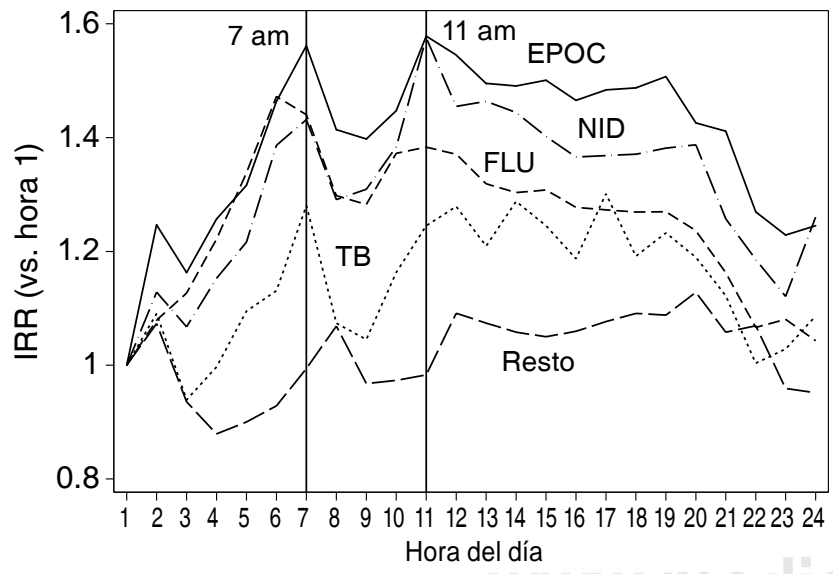

Figura 2. Muertes por hora del día, modelos de Poisson. Se muestran los cocientes de incidencia (IRR) del número de muertes de acuerdo a la hora del día. En general son similares a los descritos en la figura 1, con picos a las 7 a.m. y 11 a.m. para varias de las enfermedades, pero no para muertes perinatales. Los picos más amplios se observan para EPOC pero también para otras enfermedades respiratorias y coinciden con las de insuficiencia cardíaca y cardiopatía hipertensiva $\mathrm{e}$ isquémica (no incluidas en la figura). poran simultáneamente las horas, los meses y los años como variables indicadoras, y por lo mismo tienen un ajuste completo de las variables exploradas.

\section{RESULTADOS}

La tabla 1 muestra el total de muertes que se evaluaron por diagnóstico. La causa menos frecuente de las

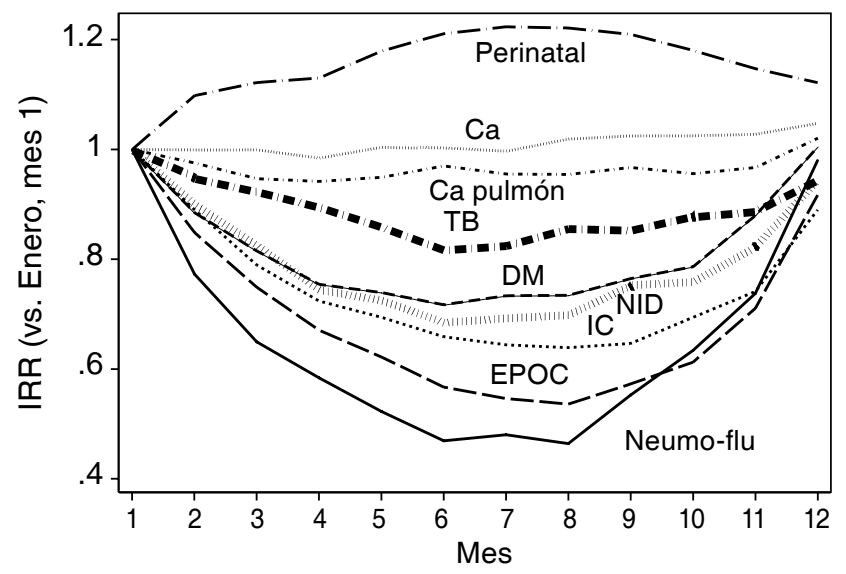

Figura 3. Muertes por mes del año, modelos de Poisson. Se muestran los cocientes de incidencia (IRR) del número de muertes de acuerdo al mes del año (comparado con enero, mes 1 al que se le asigna 1). Salvo las muertes perinatales, el máximo se obtiene en el invierno (diciembre y enero) y el mínimo en verano (julio-agosto) con las variaciones más amplias para neumonía-influenza que podrían ser el motor de la estacionalidad para las otras causas.

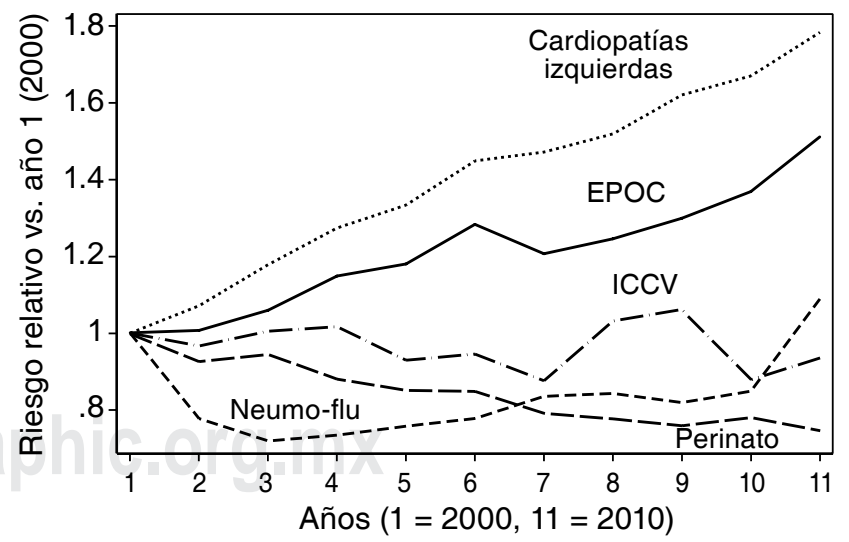

Figura 4. Muertes por año, modelos de Poisson. Se muestran los cocientes de incidencia del número de muertes de acuerdo al año de muerte, comparados con el 2000 (que se le asigna 1). El número de muertes por cardiopatías y EPOC se ha ido incrementando mientras que las perinatales han ido disminuyendo. 
muertes analizadas fue la tuberculosis con un total de 30,733 y las enfermedades restantes fueron las más numerosas con 2.66 millones de un total de 5,346,186 muertes en los grupos diagnóstico estudiados. El total de horas analizadas posibles fueron 96,431. Por lo mismo, en el análisis de la tuberculosis, de las enfermedades intersticiales y del cáncer pulmonar, la base final incorpora un número alto de horas con cero muertes.
Varias de las muertes mostraron estacionalidad con incremento invernal muy similar a la de neumoníainfluenza y probablemente sean asociadas a ésta (figura 3) así como la tabla 2 (ver los IRR mínimos durante el verano). Las oscilaciones estacionales más marcadas en el número de muertes fueron por neumonía-influenza con más del doble entre el máximo y mínimo (IRR mínimo $<0.5$ en el verano), y en relaciones (cocientes) cada vez menos significativos, EPOC (<0.55), IC (<0.7),

Tabla 1. Causas de defunción evaluadas (medias y desviación estándar).

\begin{tabular}{|c|c|c|c|c|c|c|}
\hline & $N$ & Edad & DE edad & $\begin{array}{l}\text { Muertes/ } \\
\text { hora }\end{array}$ & $\begin{array}{l}\mathrm{DE}, \text { muertes/ } \\
\text { hora }\end{array}$ & $\begin{array}{c}\% \\
\text { varones }\end{array}$ \\
\hline TB & 30,733 & 56.0 & 18.4 & 1.3 & 0.6 & 66.4 \\
\hline Tumores malignos & 639,266 & 62.3 & 7.6 & 7.8 & 3.2 & 46.7 \\
\hline DM & 711,350 & 67.4 & 5.0 & 8.8 & 4.0 & 46.4 \\
\hline Cardiopatía izquierda & 582,098 & 73.8 & 6.3 & 7.4 & 3.4 & 55.3 \\
\hline Insuficiencia cardíaca & 86,739 & 77.4 & 14.0 & 1.9 & 1.1 & 43.8 \\
\hline Neumonía/influenza & 142,795 & 59.9 & 24.4 & 2.7 & 2.2 & 53.7 \\
\hline EPOC & 206,193 & 79.1 & 7.1 & 3.3 & 1.8 & 56.2 \\
\hline Enfermedades intersticiales del pulmón & 38,196 & 73.8 & 10.9 & 1.4 & 0.7 & 42.6 \\
\hline Perinatales & 177,870 & $<1$ & - & 44.4 & 333.6 & 57.7 \\
\hline Cáncer pulmonar & 71,666 & 69.2 & 10.3 & 1.8 & 0.9 & 67.1 \\
\hline Resto & $2,659,280$ & 56.3 & 5.9 & 32.0 & 83.9 & 59.7 \\
\hline
\end{tabular}

DE: desviación estándar; TB: tuberculosis, DM: diabetes mellitus; EPOC: enfermedad pulmonar obstructiva crónica.

Total de muertes con hora y fecha de defunción: 5,346,186.

Tabla 2. Asociaciones relevantes de hora, mes y año con número de fallecimientos en México en modelos de regresión de Poisson (IRR incidence rate ratio).

\begin{tabular}{|c|c|c|c|c|c|c|c|c|c|c|c|c|}
\hline Variable & Todas & Resto & $\begin{array}{l}\text { Neumonía } \\
\text { e influenza }\end{array}$ & $\begin{array}{l}\text { Cardiopatía } \\
\text { izquierda }\end{array}$ & $\begin{array}{c}\text { Insufi- } \\
\text { ciencia } \\
\text { cardíaca }\end{array}$ & $\begin{array}{c}\text { Perinata- } \\
\text { les }\end{array}$ & EPOC & TB & NID & $\begin{array}{l}\text { Ca. } \\
\text { pulmón }\end{array}$ & $\begin{array}{l}\text { Neopla- } \\
\text { sias }\end{array}$ & DM \\
\hline \multicolumn{13}{|l|}{ Hora vs. 1 AM } \\
\hline 7:00 AM & 1.08 & 0.99 & 1.44 & 1.44 & 1.55 & 1.29 & 1.56 & 1.28 & 1.43 & 1.34 & 1.34 & 1.44 \\
\hline $11: 00$ AM & 1.09 & 0.98 & 1.38 & 1.44 & 1.53 & 1.15 & 1.58 & 1.25 & 1.58 & 1.39 & 1.36 & 1.44 \\
\hline \multicolumn{13}{|l|}{ Mes vs. enero } \\
\hline Febrero & 0.94 & 0.93 & 0.77 & 0.88 & 0.89 & 1.10 & 0.85 & 0.95 & 0.90 & 0.97 & 1.00 & 0.88 \\
\hline Junio & 0.87 & 0.83 & 0.47 & 0.72 & 0.66 & 1.21 & 0.57 & 0.81 & 0.68 & 0.97 & 1.00 & 0.72 \\
\hline Julio & 0.87 & 0.84 & 0.48 & 0.73 & 0.64 & 1.22 & 0.55 & 0.82 & 0.69 & 0.95 & 1.00 & 0.73 \\
\hline Agosto & 0.87 & 0.84 & 0.46 & 0.73 & 0.64 & 1.22 & 0.54 & 0.85 & 0.70 & 0.95 & 1.02 & 0.73 \\
\hline Diciembre & 1.02 & 1.01 & 0.98 & 1.00 & 0.89 & 1.12 & 0.92 & 0.94 & 0.94 & 1.02 & 1.05 & 1.00 \\
\hline \multicolumn{13}{|l|}{ Año vs. 2000} \\
\hline 2008 & 1.22 & 1.20 & 0.82 & 1.62 & 1.06 & 0.76 & 1.30 & 0.73 & 1.72 & 1.07 & 1.25 & 1.62 \\
\hline 2009 & 1.27 & 1.27 & 0.85 & 1.67 & 0.88 & 0.78 & 1.37 & 0.69 & 1.84 & 1.06 & 1.29 & 1.67 \\
\hline 2010 & 1.33 & 1.32 & 1.09 & 1.78 & 0.94 & 0.75 & 1.51 & 0.75 & 2.04 & 1.08 & 1.33 & 1.78 \\
\hline
\end{tabular}

El modelo de Poisson incorporaba variables indicadoras para las horas del día, meses del año y años estudiados. Se ajustó para cada una de las enfermedades estudiadas. IRR es el cociente de tasas de incidencia. Todos los coeficientes del modelo resultaron estadísticamente significativos $(p<0.05)$ y tienen un error estándar muy pequeño, debido al gran número de observaciones, $>96,000$ por cada modelo. La tabla no incluye el total de las horas, meses o años estudiados, pero sí se incorporaron al modelo. Abreviaturas: EPOC = Enfermedad pulmonar obstructiva crónica; TB = Tuberculosis; NID = Neumopatía intersticial difusa; DM = Diabetes mellitus. En los métodos pueden consultarse los códigos CIE que se incluyeron en cada categoría. 
cardiopatías izquierdas $(<0.75)$, lo que puede sugerir también una asociación con la influenza.

Los modelos de Poisson muestran también asociaciones significativas entre el número de muertos y las horas del día (tabla 2, figuras 1 y 2). Las muertes por neumonía e influenza, insuficiencia cardíaca, cardiopatías izquierdas y EPOC tuvieron un ciclo circadiano, con máximos de muertes entre las 7 y las 11 a.m.

Las oscilaciones de las muertes perinatales son mucho menores en las 24 horas (diferencias máximas de IRR de < 1.3) mientras que las de IC y EPOC alcanzan un IRR > 1.5 (figura 2).

Varias enfermedades tuvieron un incremento significativo en el número de muertos año con año ([tabla 2 y figura 4] EPOC, resto, neumonía e influenza, cardiopatía isquémica) mientras que las muertes por Tb y las perinatales fueron disminuyendo en el período estudiado.

\section{DISCUSIÓN}

En el estudio de ritmicidad del número de muertos, se encontró un patrón estacional para varias enfermedades pero también un ritmo circadiano en varias de ellas. El incremento invernal fue más marcado para neumoníainfluenza, pero también se observó para otras muertes respiratorias como las de EPOC y muertes no respiratorias como las debidas a IC y causas cardiovasculares pero, por otro lado, las perinatales no mostraron un patrón estacional. Es probable que la influenza sea el motor del incremento invernal en la mortalidad por varias causas, aun cuando no podrían descartarse las contribuciones climáticas asociadas como el descenso en la temperatura o cambios en la humedad y en el patrón de lluvias, o a los incrementos en la contaminación atmosférica. En la medida en que la influenza genera un incremento en la certificación de muertes por variadas causas, un fenómeno conocido sería prevenible con la vacunación contra la influenza.

Por otro lado, se observa un incremento en las muertes a las 7 a.m. y a las 11 a.m. (patrón bimodal para varias de ellas) en la EPOC, la IC, la neumonía-influenza, la TB, y las muertes cardiovasculares, consistente con el incremento en urgencias cardiopulmonares descrito previamente entre las 6-12 horas. ${ }^{11,12}$ En algunos de estos casos, el horario se ha atribuido al inicio de la fibrilación ventricular, ${ }^{12}$ o bien el incremento en disparadores de eventos cardiovasculares que ocurren después de despertar y levantarse de la cama, ${ }^{10}$ como los aumentos en la presión arterial y la frecuencia cardíaca o la coagulabilidad,${ }^{14}$ quizá atribuibles a un incremento en la actividad simpática. Por otro lado, el pico matutino en muertes no se observó en un estudio previo realizado en pacientes con insuficiencia cardíaca ${ }^{9}$ pero sí en enfermos tratados con amiodarona con muerte súbita..$^{15}$ La muerte súbita por tromboembolia pulmonar ocurrió cerca del mediodía ${ }^{16}$ también. La hiperactividad simpática asociada al estado de alerta y de actividad podría generar un desbalance que podría ser fatal en personas muy frágiles o susceptibles. Por ejemplo, puede subir la presión arterial, la frecuencia cardíaca con cargas extras de trabajo ventricular y con mayor tono coronario. ${ }^{14,17}$

Es posible la presencia de artefactos y sesgos en el análisis de la hora del día. Por ejemplo, los fallecimientos de las 7 de la mañana podrían haber ocurrido durante la noche, si la persona fue encontrada muerta en la mañana. Sin embargo, el pico de las 11 a.m. ya no podría atribuirse a lo mismo, y por otro lado las temperaturas ya no son las más bajas del día, ni son las horas con más contaminación. Otro posible factor que incrementaría las muertes en la mañana podría ser el fumar los primeros cigarrillos del día. Los síntomas en EPOC así como la obstrucción en EPOC y asma se incrementan en la mañana. ${ }^{17,18}$

Aunque el número de fallecimientos analizados es muy grande, el análisis de certificados de defunción tiene sesgos y limitaciones conocidas. Es claro que muchas de las causas de muerte que se certifican están sujetas a errores y que varían con la capacitación que tenga el certificador, y con la enfermedad misma, ya que hay algunas en las cuales es más probable que aparezcan en el certificado, como los tumores malignos, que otros como la EPOC con un alto grado de subdiagnóstico. ${ }^{19}$ Analizamos también sólo la causa fundamental de padecimiento en lugar de varias causas que frecuentemente están descritas en el certificado, aunque esto se ha convertido en el procedimiento habitual.

Los factores que determinan las oscilaciones estacionales o circadianas de los fallecimientos son bastante especulativos por la ausencia de información adicional a la que viene en el certificado. No analizamos, por ejemplo, las condiciones climáticas, temperatura, humedad o contaminación que podría generar información adicional contribuyente al padecimiento. Carecemos también de información clínica de los fallecidos. No se observaron incrementos significativos de los fallecimientos durante el fin de semana en los padecimientos explorados, pero no se evaluaron los períodos típicos vacacionales.

Varios métodos para analizar las variaciones cíclicas de los fenómenos, que conforman series de tiempo no fueron incorporadas en el presente reporte como los análisis espectrales, en el ámbito de la frecuencia, ya que incorporamos variaciones amplísimas de tiempo que van de horas a años, lo cual dificulta graficarlos. Asimismo, se generaron modelos de regresión de Poisson incorporando términos con senos y cosenos (siendo 
un ciclo completo, sea día, mes o año, 360 grados), pero la información obtenida fue similar a la descrita en modelos con variables indicadoras y sin ventajas para un análisis exploratorio.

A pesar de las limitantes que debilitan la explicación a las causas de las oscilaciones cíclicas en el patrón de fallecimientos, varios patrones son orientadores y conllevan posibles implicaciones preventivas. Por ejemplo, el incremento invernal del número de fallecimientos en varias enfermedades comunes respiratorias, cardiovasculares y circulatorias, ha sido atribuido a la influenza y por lo mismo podrían ser prevenibles también con la vacunación. El enfermo que padece influenza se puede descompensar de múltiples enfermedades preexistentes o bien complicarse más fácilmente, y las muertes certificarse, por ejemplo, por eventos cardiovasculares y circulatorios o exacerbaciones de EPOC. De hecho, el incremento de muertes sobre el patrón invernal, se atribuye habitualmente a la influenza y se incrementa en los años de epidemia o pandemia reconocible.

El incremento en muertes durante las horas tempranas del día se muestra más limitado de intervenciones preventivas al desconocer con más detalle los mecanismos involucrados, exceptuando el genérico de incremento en la actividad simpática con mayor carga ventricular, arritmogénesis, actividad procoagulante y quizá daño endotelial, o también los cigarrillos matutinos en los fumadores, o la actividad física relacionada al levantarse o desayunar.

En resumen, varias de las enfermedades respiratorias, cardiovasculares y la DM, primeras causas de muerte en México, muestran una estacionalidad invernal probablemente relacionada primordialmente a la influenza, así como un incremento circadiano en las muertes matutinas descrito previamente, pero de origen poco claro.

\section{REFERENCIAS}

1. Charu V, Chowell G, Palacio-Mejia LS, et al. Mortality burden of the A/H1N1 pandemic in Mexico: a comparison of deaths and years of life lost to seasonal influenza. Clin Infect Dis 2011;53(10):985-993. doi: 10.1093/cid/cir644.

2. Cech I, Smolensky MH, Lane R, Nagata H, Takahashi Y, Morimoto T. Day-to-day and seasonal fluctuations of urban mortality in Kyoto, Japan. Int J Biometeorol 1979;23(2):89-105.

3. Cech I, Youngs K, Smolensky MH, Sargent F. Day-to-day and seasonal fluctuations of urban mortality in Houston, Texas. Int J Biometeorol 1979;23(2):77-87.

4. Frankoff HM. Circannual variation of human mortality in Texas. Prog Clin Biol Res 1990;341A:797-803.
5. Oehmisch W. [Seasonal rhythm of mortality. Mortality according to age groups and seasons]. Dtsch Gesundheitsw 1969;24(22):1046-1052.

6. Ovcharov VK, Tishuk EA. [Seasonality and population mortality]. Probl Sotsialnoi Gig Istor Med 1994;(5):19-23.

7. Peckova M, Fahrenbruch CE, Cobb LA, Hallstrom AP. Circadian variations in the occurrence of cardiac arrests: initial and repeat episodes. Circulation 1998;98(1):31-39.

8. Rogot E, Fabsitz R, Feinleib M. Daily variation in USA mortality. Am J Epidemiol 1976;103(2):198-211.

9. Carson PA, O'Connor CM, Miller AB, et al. Circadian rhythm and sudden death in heart failure: results from Prospective Randomized Amlodipine Survival Trial. J Am Coll Cardiol 2000;36(2):541-546.

10. Willich SN, Klatt S, Arntz HR. Circadian variation and triggers of acute coronary syndromes. Eur Heart J 1998;19 Suppl C:C12-C23.

11. Arntz HR, Willich SN, Stern $R$, et al. Circadian variation of cardiopulmonary disease onset in the general population: an emergency care system perspective from Berlin. Ann Emerg Med 1994;23(2):281-285.

12. Arntz HR, Willich SN, Oeff M, et al. Circadian variation of sudden cardiac death reflects age-related variability in ventricular fibrillation. Circulation 1993:88(5 Pt 1):22842289.

13. Becker R, Silvi J, Ma Fat D, L'Hours A, Laurenti R. A method for deriving leading causes of death. Bull World Health Organ 2006;84(4):297-304.

14. Chen L, Yang G. Recent advances in circadian rhythms in cardiovascular system. Front Pharmacol 2015;6:71. doi: 10.3389/fphar.2015.00071.

15. Behrens S, Ney G, Fisher SG, Fletcher RD, Franz MR, Singh SN. Effects of amiodarone on the circadian pattern of sudden cardiac death (Department of Veterans Affairs Congestive Heart Failure-Survival Trial of Antiarrhythmic Therapy). Am J Cardiol 1997;80(1):45-48.

16. Buff DD, Fleisher JM, Roca JA, Jaffri M, Wyrwinski PM. Circadian distribution of in-hospital cardiopulmonary arrests on the general medical ward. Arch Intern Med 1992;152(6):1282-1288.

17. Smolensky MH, Portaluppi F, Manfredini R, et al. Diurnal and twenty-four hour patterning of human diseases: cardiac, vascular, and respiratory diseases, conditions, and syndromes. Sleep Med Rev 2015;21:3-11. doi: 10.1016/j.smrv.2014.07.001.

18. Miravitlles $\mathrm{M}$, Worth $\mathrm{H}$, Soler-Cataluna JJ, et al. Observational study to characterise 24-hour COPD symptoms and their relationship with patient-reported outcomes: results from the ASSESS study. Respir Res 2014;15:122. doi: 10.1186/s12931-014-0122-1.

19. Bernd L, Joan BS, Michael S, et al. Determinants of underdiagnosis of COPD in National and International Surveys. Chest 2015;148(4):971-985. doi: 10.1378/ chest.14-2535.

$\bowtie$ Correspondencia:

Dr. Rogelio Pérez-Padilla

Departamento de Investigación en Tabaquismo

y EPOC, Instituto Nacional de Enfermedades

Respiratorias Ismael Cosío Villegas.

Calzada de Tlalpan Núm. 4502, colonia Sección XVI,

14080, Del. Tlalpan, Ciudad de México.

Correo electrónico: perezpad@gmail.com

Los autores declaran no tener conflicto de intereses. 